\title{
Science needs systematic replicability audits
}

\author{
Aurélien Allard ${ }^{1} \&$ Simine Vazire ${ }^{2}$
}

\begin{abstract}
The credibility revolution in social science has highlighted the importance of conducting replication studies. Despite this growing awareness, the value of direct replications is still hotly debated. In this article, we identify three main functions served by replication. We argue that replications are valuable when they target important or influential studies, when they provide a general estimate of the replicability rate of a population of published articles, and when they create incentives favoring replicable research. We therefore argue that the scientific community should organize systematic large-scale replication audits of two subsets of journals' published articles: a subset of the most-cited articles, and a subset of randomly selected articles that would provide an estimate of the replicability of the journals' articles. These replicability audits should pave the way for more general quality audits of scientific journals.
\end{abstract}

\footnotetext{
${ }^{1}$ University of Geneva, Institute Ethics History Humanities.

${ }^{2}$ Melbourne School of Psychological Sciences, University of Melbourne.
} 
In 2015, the journal Science published the results of the Reproducibility Project: Psychology (RP:P), a large-scale replication project in psychology (Open Science Collaboration, 2015). The project attempted to replicate one hundred studies published in three prestigious psychology journals in 2008. Most replication attempts were not successful. In the following years, the psychology community implemented many reforms aimed at improving the rigor of psychological science, including large-scale promotions of replications, open data, and pre-registration.

This arsenal of reforms has been strong and consistent enough to be described as 'Psychology's Renaissance" (Nelson et al., 2018). However, we can't help but feel that one major actor in the scientific process has been lagging behind in implementing reforms and promoting reproducible science. As the traditional gate-keepers of quality control, scientific journals should have been at the forefront of reforms. Yet, some of the most prestigious journals have failed to fulfill their basic responsibilities. Let's take the journal Science, for instance. The publication of the RP:P in this prestigious outlet in 2015 might have been a sign that Science valued replicability and rigor. But when a new large-scale collaboration in 2018 tried to publish another replication project, this time targeting the replicability of social science experiments published in Nature and Science (Camerer et al., 2018), both of these journals refused to publish it (Nosek, 2018). While Science had been happy to publicize other journals' failures in 2015, it and Nature refused to face their responsibility in 2018.

Science's failure to take replicability seriously is not an isolated case. Reactions of scientific journals have ranged from complete inaction to the implementation of some important reforms. For instance, despite having the lowest replicability rate of the three journals examined in the RP:P, the Journal of Personality and Social Psychology has not taken any clear measures to increase its rigor. In contrast, Psychological Science implemented a variety of innovations, including Open Science badges (Eich, 2014) and Registered Replication Reports (Lindsay, 2015). We claim, however, that even the most responsive journals have still not done enough, and have failed to produce evidence that they are providing their readers with reliable knowledge. Given the uncertain reliability of psychology findings, we argue that the scientific community should organize regular large-scale replication audits of scientific journals. While this practice would be revolutionary - even in the context of the new reforms-we believe that this action would most effectively align journals' incentives with the public interest.

We thus claim that journals should be regularly audited for replicability. To substantiate this claim, we argue that direct replications contribute to scientific knowledge in three major ways. The first function of direct replications is to assess the reliability of specific findings that are judged to be of broad importance, of high value, or particularly surprising. In this case, we conduct replications because we are interested in the effects in themselves. Second, direct replications are valuable because they provide an audit of the reliability of scientific research. In this second case, we do not replicate because we are interested in some specific effects, but because we care about the dependability of the scientific process itself. Third, replications provide incentives towards conducting minimally careful research.

We argue that these three functions are best served when regular replication projects are organized at the level of scientific journals. More precisely, regular replication projects should be organized for two subsets of scientific papers: 1) the papers that have been most cited or have received the most attention since their publication, and 2) randomly chosen articles for which the replicability rate could generalize to all of the journal's published papers in that time period.

Each of the three different functions of replications has already been identified in the literature. The first function, based on the value of knowing the replicability of specific findings, is explicitly 
defended by Lewandowsky \& Oberauer (2020) and Isager \& colleagues (2020). The second function (generalizing to the replicability of the scientific literature) is the implicit goal of large-scale systematic replication projects such as the RP:P. The third (incentivizing) function is associated with the work of Paul Smaldino and Richard McElreath (2016; See also Bruner, 2013). Our main contribution here is bringing together these three different functions, and arguing that a specific consequence follows: The necessity of coordinated, large-scale replication projects as a priority for each scientific community.

\section{The three functions of replications}

While the value of conducting replications may seem obvious, it has actually been a topic of heated debate in psychology over the last ten years (Crandall \& Sherman, 2016; Schmidt, 2009; Simons, 2014; Stroebe \& Strack, 2014; Zwaan et al., 2018). Most of the debates have focused on the distinction between direct and conceptual replications (Crandall \& Sherman, 2016; Simons, 2014). While direct replications try to copy the methods of original experiments as closely as possible ${ }^{3}$, conceptual replications try to study the same phenomenon by using different operationalizations of the key constructs. Although few deny the importance of conceptual replications, direct replications have been the focus of strong criticisms.

\section{What Direct Replications Cannot Do}

One major objection to the use of direct replications is that they do not target the right scientific object. To understand this criticism, we must distinguish between general phenomena and operationalized effects. General phenomena correspond to general facts that are assumed to hold true in the world; for instance, desirability bias is the general tendency to attribute greater credence to beliefs that reinforce one's preferences. It is expressed in a general form that can hold true across a wide range of operationalizations. Operationalized effects include a complete and precise description of the measurements used to create them. While a given operationalized effect can be seen as a particular instance of the general phenomenon of desirability bias, it does not have to be interpreted this way: we can still measure an operationalized effect without referring to more general phenomena. For instance, the general phenomenon of desirability bias can be translated into operationalized effects outlining how to measure smokers' and non-smokers' perceptions of the risks of smoking, or Democrats' and Republicans' beliefs in their own electoral success in an upcoming election. We could be interested in each of these operationalized effects purely for their own sake, or as instantiations of the generalized phenomenon of desirability bias.

General phenomena and operationalized effects differ in two ways. First, general phenomena are higher-order events that subsume a wide range of possible operationalizations. Second, as a consequence, general phenomena often lack clear boundaries, whereas operationalized effects have more clearly defined properties. It is difficult to specify which conditions need to be met for a particular event to fall under the umbrella term of desirability bias. However, operationalized effects come with a description of the methods used to create them, reducing ambiguities.

The distinction between general phenomena and operationalized effects is not meant to delineate two natural kinds; there is a continuum between the most precisely described operationalization and the most general phenomenon, with possible intermediate cases. But the blurriness of the distinction does

${ }^{3}$ Of course, there are always differences between the original experiment and replication attempts. In general, replications are considered to be direct replications as long as they vary factors that are not supposed to have any causal effect on the measured effect. For more details on the definition of direct replication, see Romero, 2019. 
not mean that it is useless. In science, we typically use operationalized effects to provide estimates or tests of general phenomena. Since operationalized effects have clear boundaries, we can hope to find agreement among scientists on their reality. Agreement on the existence of broader general phenomena, with their fuzzy boundaries, is much harder.

However, these general phenomena are what scientists are interested in; we do not conduct experiments simply to learn about experiments, but to learn about the world. And learning about the world requires going beyond clearly defined measurement set-ups to much broader ideas. Thus, making claims about general phenomena requires making inferences about the meaning of operationalized effects, and these inferences can sometimes involve quite large leaps. As a consequence of the gap between operationalized effects and general phenomena, a wide range of studies is necessary to triangulate perspectives and to gain an adequate understanding of the general phenomena themselves. This is why conceptual replications - which enable us to take these broader perspectives - are valuable.

In contrast to this goal, direct replications do not try to provide a wider perspective on a phenomenon they do not address concerns about the meaning of the operationalized effects (i.e., the validity of the inferences made from operationalized effects to general phenomena). They merely aim to establish the repeatability (i.e., reliability) of the operationalized effects themselves. By definition, they keep the same methods as the ones used in the original experiment. For instance, in 2016, a large-scale collaboration tried to replicate a specific experiment investigating ego depletion, a popular psychological paradigm which claims that self-control depletes itself quickly and deeply each time it is exercised (Hagger et al., 2016; Sripada et al., 2014). Whereas the original experiment claimed to find support for the ego depletion paradigm, the replication failed to find any evidence consistent with the original effect. And this is where the distinction between general phenomena and operationalized effects comes into play. The failed replication provided evidence against a particular operationalization of ego depletion. The precise nature of this experimental set-up is very far from the generality of the phenomenon at stake. As a consequence, the replication provided limited evidence against ego depletion itself, since different operationalizations of a general phenomenon might yield completely different results. Likewise, even when a direct replication provides strong evidence for the original effect, it cannot be said to provide much evidence for the general phenomenon - the inferential leap from the operationalized effect to the general phenomenon may not be valid. No matter what the replication result turns out to be, the cautious scientist will only make confident inferences about the reliability of the operationalized effect, not the validity of broader inferences about what it means.

In short, direct replications provide only limited information about general phenomena. They mostly provide evidence regarding a particular experimental or operational set-up. At first glance, this justifies the position of authors skeptical of the value of direct replications (Crandall \& Sherman, 2016; Stroebe $\&$ Strack, 2014). However, this overlooks the specific value of direct replications. In particular, there are three contexts in which it is crucial to estimate operationalized effects.

\section{The First Function of Replications: Testing the Reliability of Valuable Operationalized Effects}

First, some operational set-ups and methods can be particularly valuable in themselves (Lewandowsky and Oberauer, 2020; Isager et al...). If we assume that some experiments are more important than others, it can be worth trying to replicate the most valuable ones even if, in many other circumstances, exploring new domains through conceptual replications would be a better strategy. These valuable experiments might be experiments 1) whose results are highly surprising, 2) that provide convincing evidence in favor of a general phenomenon (i.e., they constitute severe tests, in the sense that there are 
few, if any, alternative explanations for the result), and/or 3) that have wide-ranging consequences (for instance, because they have practical importance). Because clever designs that provide a highly-diagnostic test of a theory are hard to come by, when such tests exist, it can be better to exploit them than to explore new ground. Exploitation, in this case, means making sure that we have an accurate estimate of the effect size produced by this experiment.

The idea that we should prioritize the replication of highly influential studies has been put forward in a recent paper by Lewandowsky and Oberauer (2020). In this paper, the authors defend the idea that we should only replicate highly-cited studies, which are arguably the most important. Their argument is based on the premise that studies vary widely in terms of quality, with only a few studies having enough value that they warrant replications, and that citation counts are a reasonable proxy for quality (Isager et al., 2021). Of course, the idea that citation counts reflect quality is controversial. In any case, there is another major reason to focus on replicating widely-cited work: since these papers tend to be more widely read, the negative impact of an unreplicable result would affect more readers.

\section{The second function of replications: setting a prior on the credibility of published research}

The case for prioritizing the replication of influential studies is thus very strong. But, contrary to what Lewandowsky and Oberauer (2020) seem to assume, this does not exhaust the value of direct replications. Even if a single replication of an average-quality experiment is of little value in itself, large-scale replication projects can give us something valuable: a general audit of the state of a field, and an estimate of the reliability of the scientific process itself. And this second function is not exhausted by the exclusive replication of the most influential papers. Indeed, it is unclear whether we should expect the replicability rate of the broader scientific literature to be higher or lower than that of the most influential papers (we can think of arguments on both sides). Thus, the question of how reliable our findings - and thus our scientific process - are as a whole cannot be answered by looking at the replicability of the most influential studies. A different process is required to ensure that we get a proper estimate of the average reliability of scientific papers.

From an epistemic point of view, replications provide feedback on the scientific process, both to the original authors themselves and to the scientific community at large. When replication studies are generalizable - i.e., when the sample of studies tested is drawn at random from a clearly-defined population - the information they provide helps to set a prior belief on the reliability of studies in the same population whose replicability has not been tested.

This feedback, or audit value, of replications means that they can have a great deal of impact beyond the simple estimates of the studies that have actually been replicated. If a researcher knows that studies in her field tend to produce exaggerated effect sizes, then she can take precautions when planning the sample size or precision necessary for her studies, by, for instance, assuming that the likely true effect size is a fraction of the published effect sizes. If reviewers and journal editors are aware that many results are unreliable, they can be more attentive to potential sources of bias such as flexible data analysis and questionable research practices. The prior that is set by replication studies produces general knowledge that has wide-ranging consequences, even when most experiments are not subjected to direct replication.

Of course, it may be too costly to try to replicate all experiments. But that doesn't mean that we can't get a good estimate of the reliability of the scientific process via random sampling. Even if none of your experiments ever get replicated, you can adjust your confidence in your own findings by taking into account the average replicability of your field. The RP:P came close to this ideal and produced a 
drastic reevaluation of our beliefs in the state of psychology, leading to widespread changes in practices $^{4}$.

\section{The third function of replications: creating incentives towards replicable research}

From an economic point of view, replication results function like incentives, rewarding careful research and punishing unreliable science. This economic function of replications will obtain if replication outcomes have an impact on the reputation of the actors (researchers, editors, professional societies) who have either conducted or promoted/endorsed the research.

At a micro-level, a high-quality replication failure should impact the reputation of the original researchers. Of course, a single replication failure should not have much of an impact, because a single replication failure can have multiple causes (most importantly, the original could have been a fluke, even in the absence of any questionable practices). However, a single high-quality replication failure should still have some (minimal) impact on reputation, and multiple replication failures should be seen as a strong signal that there is something amiss in the original research process. Most importantly, if researchers can anticipate the negative reputational consequences of replication failures, and if replication failures are taken into account for promotion and recruitment in academia, this should lead researchers to reform their own practices.

While the reputational impact on individual researchers is useful, the incentive value of replications is arguably even more important at higher levels. At a macro-level, replication failures can have an impact on the reputation of two actors: scientific journals and scientific communities. If a journal suffers repeated replication failures, then it would be a sure sign that their published articles should be read with skepticism, which in turn should give them incentives to be more cautious regarding the articles they publish in the future. If a scientific field suffers from replication failures, then it should push these researchers to collectively organize to improve their scientific practices. This was the meaning of, for instance, Daniel Kahneman's open letter to the researchers involved in priming research (Yong, 2012), where he urged priming researchers to reform their practices to preserve their reputation.

Reputational incentives faced by macro-actors will have the most positive benefits if these actors have the possibility of influencing in turn the behavior of individual researchers. This is clearest in the case of scientific journals. If journals require higher levels of methodological quality for article acceptance, then it seems very likely that researchers would design their future studies to fulfill these expectations. Likewise, if universities start taking into account replicability for recruitment practices, or professional societies select awardees and keynote speakers based in part on their track records with respect to replicability, this will help shape individual researchers' behavior. In the next section, we will argue that replications projects should be conducted at the level of journals, in order to create competition among them and ultimately change the academic incentive structure.

\section{The need for systematic audits of academic journals}

Organizing replication research is currently at the discretion of self-organized groups of researchers. Here we argue that a more systematic approach is needed: scientific journals should be regularly

\footnotetext{
${ }^{4}$ Admittedly, the sampling of studies in the RP:P was not truly random, providing some uncertainty in how representative the selected sample was. For debates regarding this issue, see Gilbert et al., 2016; Anderson et al., 2016.
} 
audited on their replicability. This focus on scientific journals stems from three main reasons. First, journals often advertise themselves as providing quality control (through peer review and editorial oversight), but this claim is rarely put to any empirical test. Quality control can mean many things, but it is hard to deny that moderate-to-high levels of replicability should be one of the minimum features of high-quality science. Replicability is not sufficient for quality, but it is necessary. Thus, if they want to be consistent with their proclaimed goals, journals should ensure that the replicability rate of their published articles is high, or, if they are satisfied with a low replicability rate, that they advertise themselves in a way that is consistent with this (and accept the reputational consequences).

Second, journals are the principal agents who set incentives within the academic system, and no science can flourish if the incentives are stacked against replicability. The academic career is based on publications in prestigious journals, and academics respond to incentives created by journals. If journals do not know whether their own articles are replicable (i.e., if they don't know whether they are successfully selecting for reliability in their editorial decisions), it is impossible for them to know whether they are setting the right incentives.

Third, organizing replications journal-wise can create a virtuous competition among the main actors in a scientific field. Currently, the only common competitive measure among scientific journals is the journal impact factor, which encourages the selection of irreproducible science as long as sexy but implausible papers are likely to attract attention (Smaldino \& McElreath, 2016). We need to set other incentives by which journals could distinguish themselves from their competitors (e.g., Fraley \& Vazire, 2014; TOP Factor, 2020). Ranking journals based on replicability would be another way of aligning the incentives with the common good. Ideally, other rankings based on evaluations of validity (not just reliability) would also be developed and contribute to virtuous competition among journals. Despite how revolutionary our proposal may sound in the current climate, replicability is actually a rather unambitious goal to strive for.

If we accept the premises of our arguments, what practical changes follow? Who should organize these journal-wise replication projects? In our opinion, scientific journals themselves have a moral duty to organize these audits, since they need them to know whether they are performing their role of gatekeepers of the scientific process correctly. In this context, journals could, every four or five years, select $10 \%$ of their papers that have received the most citations, and a random $10 \%$ of all their papers. Among the $10 \%$ of the most important papers, the most important study within the paper should be selected for replication. Among the random 10\%, a random study should be selected. While it is certainly tricky to estimate the right proportion of original studies that should be replicated, we think that replicating such a small subset of studies should not be too massive a burden in terms of time and resources. Of course even a modest burden can seem insurmountable in an environment where resources are already spread thin. However, the allocation of resources reflects a community's values, and if a scientific community is not willing to invest some of its resources into verifying the replicability of its body of knowledge, we wonder whether it can claim to be committed to the core scientific value of self-correction.

Here is a concrete way to implement this proposal: journals could select the to-be-replicated articles, and then publish an open call for replications in the form of Registered Reports (Chambers, 2013). The oversight of these replication projects would be delegated to an external editor, in order to minimize conflicts of interests. The journals would commit to publishing these Registered Replication Reports, regardless of the results. Ideally, journals would also fund these replication attempts, but this might not be feasible for journals that have no financial revenue stream (e.g., through subscriptions or high 
Article Processing Charges). In the long run, funding agencies should take into account this function of scientific journals and provide support for this type of replication project.

While the organization of replicability audits by scientific journals is probably the most realistic option right now, it is arguably not an ideal solution, due to the presence of possible conflicts of interest. In the long-run, alternative replication schemes could be envisaged. For instance, a scientific society could be constituted to complete these replication projects. Felipe Romero, in a series of recent papers, has also promoted the recruitment of professional replicators as a separate academic track (Romero, $2018,2020)$. If such a scheme were to succeed, then these professional replicators would be ideally positioned to conduct these audits. While such alternative schemes would be ideal in the long run, we think that, in the current context, replication audits are most likely to succeed if they are promoted by journals themselves, for two main reasons. First, the endorsement of audits by journals would lend legitimacy to the project. Second, journals can offer strong incentives for replications, since they can promise to publish any replication attempt conducted by the auditing teams.

\section{Why focus on replicability?}

We have so far focused on the importance of replicability for a healthy science. We do not want to suggest, however, that replicability is the most important sign of quality in research. For instance, every undergraduate student in psychology learns that a high-quality study has to be valid, often broken down into the "four validities" (Shadish, Cook, \& Campbell, 2002): construct validity, internal validity, external validity, and statistical validity. In themselves, direct replications only assess one narrow aspect of statistical validity (the rate of false positives and negatives), and say nothing about the other kinds of validity. However, replicability audits are important not because replicability is a sufficient condition for high-quality science but because replicability is a necessary condition, and one that is easier to evaluate than other characteristics of scientific validity.

The idea that replicability is a necessary condition for high-quality science does not seem controversial. If a particular study claims to show a wonderfully interesting new effect, but the effect cannot be found in high-quality replication attempts, we are almost certainly going to put a low value on the original experiment.

To substantiate the claim that replicability audits are easier to interpret than other evaluative practices, consider how different quality audits could be performed with different kinds of validity criteria. We could focus on auditing external validity, for instance; this could take the form of conducting crosscultural replication studies to assess whether our experiments still hold in a vast array of human cultures, or replicating studies with different operationalizations of the constructs of interest (Landy et al., 2020). Auditing internal validity could be implemented by asking large teams of scientists to propose alternative explanations for the effect, and having them test these alternative explanations. Auditing construct validity could take the form of running validation studies on the measures and manipulations used in the published articles.

It is clear that these auditing practices could have a tremendous positive impact on current research practices in psychology. In the long run, we believe that the field would benefit from conducting a wide range of such audits. But these quality checks come with an additional degree of complexity and ambiguity. Probing external validity by conducting replications in different cultures or contexts may prove to be much more onerous than the simple replication of the original study. Assessing the 
construct validity of measures lacks the simple interpretability of the assessment of replication outcomes. Replications offer a promising area for audits, in that their interpretation is comparatively simple (see, e.g., Simonsohn, 2015 for a clear discussion of how to interpret replication outcomes). The fact that the effect sizes in replication studies have consistently been about half as large as the original effect sizes in major psychology journals is, in itself, a major testimony of the failures of the field, and a fact that can be immediately acted upon. Any journal taking its reputation seriously should launch reforms when faced with such failures. Given that similarly serious concerns have been raised about other aspects of statistical validity (e.g., Nuijten, 2016), external validity (Yarkoni, 2019), internal validity (Flannelly et al., 2018), and construct validity (Flake et al., 2017), we fully support the development of auditing mechanisms for all of these, and other, aspects of scientific rigor. However, we believe this will prove to be difficult, and should not delay action on the much more tractable problem of replicability. In our mind, replication audits should thus be seen as a stepping stone towards broader assessments of quality in psychology journals. Once we accept the idea that post-publication scrutiny is an essential part of a flourishing field, we can build on this cultural change to promote higher quality standards in other areas as well (Vazire et al., 2020).

\section{Conclusion: taking replicability seriously, at $\mathrm{l}(\mathrm{e})$ ast}

We have highlighted three functions of direct replications. First, replications provide an estimate of an operationalized effect. Since the value of this type of replication depends on the value of the original study itself, this first kind of replication should target the most important experiments. Second, replications provide an evaluation of the scientific process itself. This second function can be best realized via randomized evaluations of the scientific literature. Third, replications provide incentives towards conducting replicable research. These three functions yield two kinds of duties for scientific journals: a duty to organize replications of their most-cited experiments, and a duty to replicate a random subset of their published studies.

Our proposal is based on a simple discordance. In the last ten years, replicability projects have repeatedly shown that journals are failing in their duty to guarantee the publication of mostly reliable science. Despite the mounting evidence regarding the failures of scientific journals, the actual task of conducting replications has mostly been left to the independent efforts of individual scientists or grassroots collaborations. But replications are necessary for journals to know whether they are betraying their mission of offering reliable knowledge regarding the world. We hope that journals will, at last, take seriously their duty of ensuring the quality of published research. And what's more: we hope that, in the future, replicability rates will become a new way of judging the quality of a journal, and pave the way for broader and more systematic audits of the quality of research articles.

\section{Author Contributions}

Both authors jointly generated the idea for the article. The first author wrote the first draft of the manuscript, and both authors critically edited it. Both authors approved the final submitted version of the manuscript.

\section{Acknowledgments}

The authors wish to thank Jessie Sun, Julia Bottesini, Alex Holcombe, Dan Simons, and Brian Nosek for their feedback on previous versions of this manuscript. 


\section{References}

Anderson, C. J., Bahník, Š., Barnett-Cowan, M., Bosco, F. A., Chandler, J., Chartier, C. R., Cheung, F., Christopherson, C. D., Cordes, A., Cremata, E. J., Penna, N. D., Estel, V., Fedor, A., Fitneva, S. A., Frank, M. C., Grange, J. A., Hartshorne, J. K., Hasselman, F., Henninger, F., ... Zuni, K. (2016). Response to Comment on "Estimating the reproducibility of psychological science." Science, 351(6277), 1037-1037. https://doi.org/10.1126/science.aad9163

Bruner, J. P. (2013). Policing epistemic communities. Episteme, 10(4), 403-416. https://doi.org/10.1017/epi.2013.34

Camerer, C. F., Dreber, A., Holzmeister, F., Ho, T.-H., Huber, J., Johannesson, M., Kirchler, M., Nave, G., Nosek, B. A., Pfeiffer, T., Altmejd, A., Buttrick, N., Chan, T., Chen, Y., Forsell, E., Gampa, A., Heikensten, E., Hummer, L., Imai, T., ... Wu, H. (2018). Evaluating the replicability of social science experiments in Nature and Science between 2010 and 2015. Nature Human Behaviour, 2(9), 637-644. https://doi.org/10.1038/s41562-018-0399-Z

Chambers, C. D. (2013). Registered Reports: A new publishing initiative at Cortex. Cortex: A Journal Devoted to the Study of the Nervous System and Behavior, 49(3), 609-610. https://doi.org/10.1016/j.cortex.2012.12.016

Collaboration, O. S. (2015). Estimating the reproducibility of psychological science. Science, 349(6251). https://doi.org/10.1126/science.aac4716

Crandall, C. S., \& Sherman, J. W. (2016). On the scientific superiority of conceptual replications for scientific progress. Journal of Experimental Social Psychology, 66, 93-99. https://doi.org/10.1016/j.jesp.2015.10.002

Eich, E. (2014). Business Not as Usual. Psychological Science, 25(1), 3-6. https://doi.org/10.1177/0956797613512465

Flake, J. K., Pek, J., \& Hehman, E. (2017). Construct Validation in Social and Personality Research: Current Practice and Recommendations. Social Psychological and Personality Science, 8(4), 370-378. https://doi.org/10.1177/1948550617693063

Flannelly, K. J., Flannelly, L. T., \& Jankowski, K. R. B. (2018). Threats to the Internal Validity of Experimental and Quasi-Experimental Research in Healthcare. Journal of Health Care Chaplaincy, 24(3), 107-130. https://doi.org/10.1080/08854726.2017.1421019 
Fraley, R. C., \& Vazire, S. (2014). The N-Pact Factor: Evaluating the Quality of Empirical Journals with Respect to Sample Size and Statistical Power. PLOS ONE, 9(10), e109019. https://doi.org/10.1371/journal.pone.0109019

Gilbert, D. T., King, G., Pettigrew, S., \& Wilson, T. D. (2016). Comment on "Estimating the reproducibility of psychological science." Science, 351(6277), 1037-1037. https://doi.org/10.1126/science.aad7243

Hagger, M. S., Chatzisarantis, N. L. D., Alberts, H., Anggono, C. O., Batailler, C., Birt, A. R., Brand, R., Brandt, M. J., Brewer, G., Bruyneel, S., Calvillo, D. P., Campbell, W. K., Cannon, P. R., Carlucci, M., Carruth, N. P., Cheung, T., Crowell, A., De Ridder, D. T. D., Dewitte, S., ... Zwienenberg, M. (2016). A Multilab Preregistered Replication of the Ego-Depletion Effect. Perspectives on Psychological Science, 11(4), 546-573. https://doi.org/10.1177/1745691616652873

Isager, P. M., Veer, A. van 't, \& Lakens, D. (2021). Replication value as a function of citation impact and sample size. MetaArXiv. https://doi.org/10.31222/osf.io/knjea

Isager, Peder, van Aert, Robbie, Bahník, Štěpán, Brandt, Mark, DeSoto, Kurt, Giner-Sorolla, Roger, Krueger, Joachim, Perugini, Marco, Ropovik, Ivan, van 't Veer, Anna, Vranka, Marek, \& Lakens, Daniel. (2020). Deciding what to replicate. https://doi.org/10.17605/OSF.IO/ASYPE

Landy, J. F., Jia, M. L., Ding, I. L., Viganola, D., Tierney, W., Dreber, A., Johannesson, M., Pfeiffer, T., Ebersole, C. R., Gronau, Q. F., Ly, A., van den Bergh, D., Marsman, M., Derks, K., Wagenmakers, E.J., Proctor, A., Bartels, D. M., Bauman, C. W., Brady, W. J., ... Uhlmann, E. L. (2020). Crowdsourcing hypothesis tests: Making transparent how design choices shape research results. Psychological Bulletin, 146(5), 451-479. https://doi.org/10.1037/bul0000220

Lewandowsky, S., \& Oberauer, K. (2020). Low replicability can support robust and efficient science. $N a$ ture Communications, 11(1), 1-12. https://doi.org/10.1038/s41467-019-14203-0

Lindsay, D. S. (2015). Replication in Psychological Science. Psychological Science, 26(12), 1827-1832. https://doi.org/10.1177/0956797615616374

Nelson, L. D., Simmons, J., \& Simonsohn, U. (2018). Psychology’s Renaissance. Annual Review of Psychology, 69(1), 511-534. https://doi.org/10.1146/annurev-psych-122216-011836

Nosek, B. (2018, August 29). I have been asked many times if we submitted the Social Sciences Replication Project to Science and Nature first. Twitter. https://twitter.com/BrianNosek/sta$\underline{\text { tus/1034792697247289344 }}$

Nuijten, M. B., Hartgerink, C. H. J., van Assen, M. A. L. M., Epskamp, S., \& Wicherts, J. M. (2016). The prevalence of statistical reporting errors in psychology (1985-2013). Behavior Research Methods, 48(4), 1205-1226. https://doi.org/10.3758/s13428-015-0664-2 
Romero, F. (2018). Who Should Do Replication Labor? Advances in Methods and Practices in Psychological Science, 1(4), 516-537. https://doi.org/10.1177/2515245918803619

Romero, F. (2019). Philosophy of science and the replicability crisis. Philosophy Compass, 14(11), e12633. https://doi.org/10.1111/phc3.12633

Romero, F. (2020). The Division of Replication Labor. Philosophy of Science, 87(5), 1014-1025. https://doi.org/10.1086/710625

Schmidt, S. (2009). Shall we Really do it Again? The Powerful Concept of Replication is Neglected in the Social Sciences. Review of General Psychology, 13(2), 90-100. https://doi.org/10.1037/a0015108

Simons, D. J. (2014). The Value of Direct Replication. Perspectives on Psychological Science, 9(1), 76-80. https://doi.org/10.1177/1745691613514755

Simonsohn, U. (2015). Small Telescopes: Detectability and the Evaluation of Replication Results. Psychological Science. https://doi.org/10.1177/0956797614567341

Smaldino, P. E., \& McElreath, R. (2016). The natural selection of bad science. Royal Society Open Science, 3(9), 160384. https://doi.org/10.1098/rsos.160384

Sripada, C., Kessler, D., \& Jonides, J. (2014). Methylphenidate Blocks Effort-Induced Depletion of Regulatory Control in Healthy Volunteers: Psychological Science. https://doi.org/10.1177/0956797614526415

Stroebe, W., \& Strack, F. (2014). The Alleged Crisis and the Illusion of Exact Replication. Perspectives on Psychological Science, 9(1), 59-71. https://doi.org/10.1177/1745691613514450

TOP Scores. (n.d.). Retrieved June 18, 2020, from https://topfactor.org/

Vazire, S., Schiavone, S. R., \& Bottesini, J. G. (2020). Credibility Beyond Replicability: Improving the Four Validities in Psychological Science. PsyArXiv. https://doi.org/10.31234/osf.io/bu4d3

Yarkoni, T. (2019). The Generalizability Crisis [Preprint]. PsyArXiv. https://doi.org/10.31234/osf.io/jqw35

Yong, E. (2012). Nobel laureate challenges psychologists to clean up their act. Nature. https://doi.org/10.1038/nature.2012.11535

Zwaan, R. A., Etz, A., Lucas, R. E., \& Donnellan, M. B. (2018). Making replication mainstream. Behavioral and Brain Sciences, 41. https://doi.org/10.1017/S0140525X17001972 